\title{
Proteção de Redes Ópticas Elásticas com Multiplexação Espacial Baseada em Agregação de Tráfego, Sobreposição de Espectro e Compartilhamento de Caminho de Backup
}

\author{
Helder M. N. da S. Oliveira ${ }^{1}$, Nelson L. S. da Fonseca ${ }^{1}$ \\ ${ }^{1}$ Instituto de Computação - Universidade Estadual de Campinas (UNICAMP) \\ Campinas 13083-852, SP, Brasil \\ helderalrc.ic.unicamp.br, nfonsecalic.unicamp.br
}

\begin{abstract}
The introduction of space division multiplexing (SDM) in optical networks brings new challenges for network protection, since a light path can span multiple cores. In this article, we investigate the problem of dynamic protection against simple faults in optical networks using spatial division multiplexing. For this, we propose a new path protection algorithm by performing optical traffic grooming and spectrum overlap. In the Spectrum, Backup, Routing and Core assignment (SBRC) algorithm, each connection is assigned to a primary path and a backup path.
\end{abstract}

Resumo. A introdução da multiplexação por divisão espacial (SDM) em redes ópticas traz novos desafios para proteção de redes, uma vez que um caminho de luz pode abranger múltiplos núcleos. Neste artigo, investiga-se o problema da proteção dinâmica contra falhas simples em redes ópticas utilizando multiplexação espacial. Para isso, propomos um novo algoritmo para proteção de caminho realizando agregação de tráfego óptica e sobreposição de espectro. No algoritmo Spectrum, Backup, Routing and Core assignment (SBRC), cada conexão é atribuída a um caminho primário e a um caminho de backup.

\section{Introdução}

As redes ópticas são redes de comunicação de dados que operam em altíssima velocidade e utilizam a luz para transmissão de dados. Estas redes possuem baixa latência e permitem o uso de grande quantidade de banda passante, permitindo o crescimento da Internet. A implementação da redes ópticas atuais é baseada em comutação de circuitos, apesar desta comutação ainda ser implementada eletronicamente. Essas redes oferecem circuitos dedicados para seus clientes, disponibilizando banda passante segura e dedicada. Contudo, são ineficientes na utilização dos recursos da rede, que ficam ociosos quando um usuário não transmite dados. A evolução das fibras ópticas teve seu marco inicial com o desenvolvimento da tecnologia WDM (Wavelength Division Multiplexing). Nessa tecnologia, a largura de banda de uma fibra óptica é dividida em um certo número de comprimento de ondas, cada um dos quais possui alta taxa de transmissão. Com o intuito de diminuir os recursos ociosos decorrentes do uso da grade fixa das redes WDM, as redes ópticas elásticas foram propostas. Sua principal vantagem é a capacidade de transmitir dados em diferentes taxas, alocando o espectro óptico de acordo com a demanda, garantindo, assim, uma alta eficiência espectral. No entanto, a capacidade de fibras ópticas de um único núcleo está chegando ao seu limite, o que tem motivado a exploração da dimensão espacial (SDM). 
Embora algoritmos para alocação de espectro e núcleo tenham sido propostos [Huang et al. 2016, Tode and Hirota 2014, Muhammad et al. 2014b, Oliveira and da Fonseca 2016a], nenhum outro estudo relacionado à proteção em redes ópticas elásticas utilizando compartilhamento de caminhos de backup, agregação de tráfego óptico sobreposição de espectro foi proposto até então. A proteção é de suma importância nas redes de transporte óptico pois estas transportam grande quantidade de tráfego. Dada a enorme capacidade de uma fibra óptica empregando SDM, qualquer interrupção implica em enorme perdas de dados. À medida que o tráfego transportado aumenta, a necessidade de adoção de esquemas de proteção eficiente também aumenta. Essa vulnerabilidade motivou o desenvolvimento de diferentes esquemas de proteção e restauração para redes ópticas elásticas que utilizam fibra de núcleo único (SCF).

A proteção de caminho de backup compartilhado (SBPP) é uma técnica de proteção que tem sido intensamente investigada na última década devido ao compartilhamento eficiente de capacidade disponível e flexibilidade no provisionamento de serviços. Em redes ópticas elásticas que empregam mutiplexação espacial, o SBPP emprega um esquema de proteção 1: $\mathrm{N}$ em que os caminhos de backup podem usar o mesmo conjunto de espectro (slots), desde que seus caminhos primários correspondentes sejam enlaces disjuntos.

Em redes ópticas elásticas, a agregação de tráfego é uma técnica que combina múltiplas conexões em um caminho óptico sem necessidade de bandas de guarda entre elas [Zhang et al. 2012]. O espectro de compartilhamento é uma técnica na qual dois caminhos de luz de backup podem usar os mesmos núcleos, enlace e espectro, uma vez que os caminhos primários das duas conexões são fisicamente disjuntos [Liu et al. 2013]. A combinação de agregação de tráfego e compartilhamento de espectro permite um aumento significativo na utilização do espectro, o que diminui o bloqueio de conexões.

Apesar de outros trabalhos tratarem de proteção em redes óptica elásticas com multiplexação espacial [Oliveira and da Fonseca 2017c, Oliveira and da Fonseca 2017a], nenhum dos trabalhos na literatura empregam agregação de tráfego, caminhos de backup compartilhados e sobreposição de espectro para proteção de caminhos em redes óptica com multiplexação espacial. Este artigo introduz o algoritmo Spectrum, Backup, Routing and Core assignment (SBRC) para prover proteção para redes ópticas elásticas com multiplexação espacial. O algoritmo SBRC estende o algoritmo SBPPMC [Oliveira and da Fonseca 2017a] para utilizar a técnica de sobreposição do espectro nos caminhos ópticos de backup. $\mathrm{O}$ algoritmo decide sobre caminhos de proteção, utilizando caminhos compartilhados, agregação de tráfego e sobreposição de espectro. O algoritmo SBRC prioriza o compartilhamento e reduz o número de bloqueio de conexões.

Este artigo está organizado da seguinte forma. A seção 2 apresenta as redes ópticas elasticas com multiplexação espacial. A seção 3 revisa trabalhos relacionados. A seção 4 introduz o algoritmo SBRC. A seção 5 avalia o desempenho do algoritmo proposto e a seção 6 conclui o artigo.

\section{Redes Ópticas Elásticas com Multiplexação Espacial}

As redes ópticas elástica (EON), usam duas dimensões, o tempo e a frequência, para fornecer recursos de redes ópticas com demanda de tráfego variável. Atualmente, estas redes exploram uma terceira dimensão, o espaço, ou seja a multiplexação por divisão de espaço, SDM, melhorando significativamente o potencial da capacidade de transmissões 
futuras e a eficiência espectral das redes [Proietti et al. 2015]. A técnica de múltiplos núcleos de fibra (Multi-core fibers, MCF) é uma forma eficiente de concepção e fabricação para utilização de SDM. A adoção destas fibras ópticas aumenta a capacidade das fibras por um fator $\mathrm{C}$, onde $\mathrm{C}$ é o número de cores dentro da fibra.

Além dos efeitos físicos presentes em EON (acumulação de ruído óptico, mistura de quatro ondas), a dimensão espacial pode introduzir uma quantidade significativa de interferência de banda, devido ao modo de acoplamento e crosstalk (XT) nos dispositivos multiplexadores/demultiplexadores (MUX/DEMUX) espaciais distribuídas ao longo dos enlaces de fibra.

Em redes WDM e EON, quando uma requisição de largura de banda chega a um nó, a rede tem de alocar uma parte do espectro (alocação de recursos) para esta requisição e encontrar um caminho físico disponível para o nó destino (roteamento). Com a introdução da dimensão do espaço, esta operação torna-se mais flexível; no entanto, aumenta a complexidade de encaminhamento e alocação. Este problema é denominado de Roteamento e alocação de núcleo e espectro, (Routing, spectrum and core allocation, RSCA). Estes métodos gerenciam o roteamento na rede SDM e alocam a largura de banda disponível, em diferentes núcleos de MCFs, levando também em consideração o crosstalk entre núcleos [Fujii et al. 2013], [Muhammad et al. 2014b] e [Muhammad et al. 2014a].

Atualmente as abordagens de SDM para fibra óptica variam para diferentes tecnologias, tais como: few-mode fibers (FMFs), multimode fibers (MMF), multi-core fibers (MCFs) e coupled-core fibers (CCFs). A Figura 3 ilustra esquemas de representação para redes ópticas com multiplexação espacial.

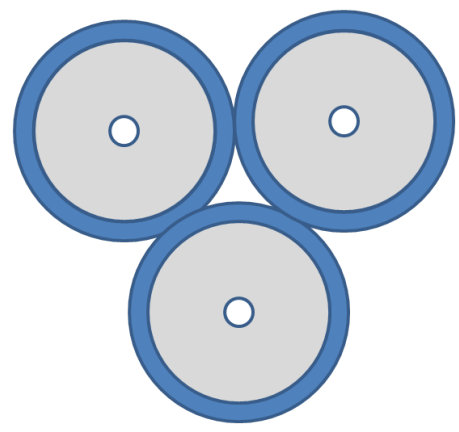

1.1 Multiplas SMF

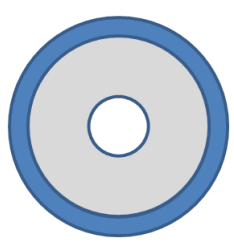

1.2 FMFs

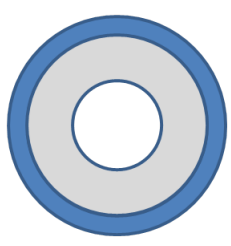

$1.3 \mathrm{MMF}$

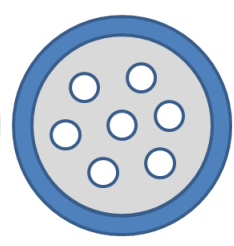

$1.4 \mathrm{MCFs}$

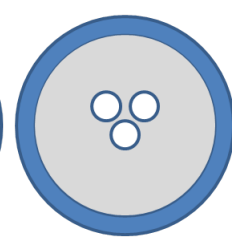

$1.5 \mathrm{CCFs}$

Figura 1. Esquemas de representação para redes ópticas SDM

Os requisitos para uma fibra com poucos modos (FMFs) são semelhantes aos das fibras de transmissão monomodo: baixa atenuação, pequena não linearidade e um elevado coeficiente de dispersão. Além disso, as fibras devem suportar um número específico de modos e baixo acoplamento entre os modos [Grüner-Nielsen et al. 2012, Sillard et al. 2014, Mori et al. 2014].

As fibras multimodo (MMF) seguem a recomendação ITU-T. Nela, é descrito que um cabo de fibra óptica multimodal possui índice graduado de 50/125 $\mu \mathrm{m}$. Em MMF, o número de modos suportados por uma fibra depende do tamanho do núcleo e do índice de refração do revestimento da fibra.

Takara et.al. [Takara et al. 2012] realizaram experimentos utilizando fibras de múltiplos núcleos (MCFs) e demonstraram a grande capacidade de transmissão dessas 
fibras. É demonstrado também que aumentando a capacidade de transmissão de MCF, aumenta-se a eficiência espectral, definida como um produto do número de núcleos e da eficiência espectral por núcleo. O crosstalk também é analisado. Em MCF, cada núcleo atua como uma fibra de único modo.

A fibras com núcleos acoplados (CCFs) [Ryf et al. 2012], consistem em 3 núcleos de modo único uniformemente espaçados em um rebordo padrão de $125 \mu \mathrm{m}$ de diâmetro.

Atualmente, a melhor solução de multiplexação espacial não é definida. A escolha da melhor solução não depende apenas do meio de fibra, mas também de componentes ópticos como (de) multiplexadores, amplificadores, filtros ópticos e switches. Existem duas abordagens para subdividir os tipos de fibras: multimodo e múltiplos núcleo, na qual os modos de fibra ortogonal ou núcleos espaciais ortogonais são utilizados como canais de transmissão. O primeiro tipo de fibra compreende o FMF e MMF, e o último tipo de fibra compreende CCFs e MCFs. Neste trabalho, consideramos a abordagem MCFs devido as suas vantagens: (i)menor crosstalk, independência entre núcleos e maior custo beneficio.

O estudo de fibras de múltiplos núcleos para multiplexação por divisão espacial, vem analisando diversas questões, tais como: alocação eficiente dos núcleos para reduzir o crosstalk e definição de características para espessura do revestimento [Hayashi et al. 2011, Ito et al. 2013, Sakaguchi et al. 2013, Saridis et al. 2015, Takenaga et al. 2011]. Obviamente, o desafio é juntar tantos núcleos quanto possível em uma única estrutura de fibra, evitando grandes penalidades de interferências inter-core.

No contexto de redes ópticas com multiplexação espacial, o crosstalk é a interferência indesejada que um núcleo de um enlace de transmissão causa em outro núcleo do mesmo enlace, ou seja, a relação entre a potência óptica inserida pelos núcleos adjacentes e em um núcleo especifico, dividida pela potência do sinal já nesse núcleo e medida em $\mathrm{dB}$. O limite, além do qual a integridade do sinal é alterada, pode variar entre $-16 \mathrm{~dB}$ e $-32 \mathrm{~dB}$, dependendo do formato de modulação que é usado [Saridis et al. 2015, Winzer et al. 2011].

Nas simulações deste artigo, foi usado um limite de cerca de $-32 \mathrm{~dB}$. Para calcular o crosstalk (XT) de um núcleo com $n$ núcleos vizinhos ativos de um MCF homogêneo, foi utilizada a fórmula baseada da Eq. 1 [Hayashi et al. 2011, Hayashi et al. 2012, Saridis et al. 2015], que também considera a teoria do poder acoplado [Koshiba et al. 2011], levando à Eq. 2. A Equação 2 será utilizada, neste artigo, para garantir a disponibilidade das conexões, com relação aos núcleos vizinhos.

$$
h=\frac{2 \cdot k^{2} \cdot R}{\beta \cdot D}
$$

Na Eq. 1, $h$ é o aumento da interferência média por unidade de comprimento, calculado por vários parâmetros da fibra: $k, \beta, R, D$, são, respectivamente, o coeficiente de acoplamento, a constante de propagação, o raio de curvatura e a distancia entre os núcleos.

$$
X T=\frac{n\{1-\exp (-(n+1) \cdot 2 \cdot h \cdot L\}}{1+n\{\exp (-(n+1) \cdot 2 \cdot h \cdot L)\}}
$$


A Eq. 2 faz uso de $h$ da equação 1 multiplicada por $L$ que é o comprimento da fibra, enquanto $n$ representa o número de núcleos adjacentes.

Neste artigo, assume-se um valor máximo de $n=6$, Além disso, os outros parâmetros assumiram valores realísticos, sendo $k=2 \times 10^{-5}, R=50 \mathrm{~mm}, \beta=4 \times 10^{6}$ e $D$ $=45 \mu \mathrm{m}$. O pior caso de crosstalk para os núcleos sempre será o núcleo central (ou qualquer outro núcleo que tenha o maior número de núcleos vizinhos), uma vez que recebe interferência indesejada de todos os seus núcleos adjacentes. Enfatiza-se que o crosstalk inter-núcleo ocorre apenas entre os mesmos slots de frequência usados em núcleos adjacentes. Nas simulações, assume-se que o espectro de cada núcleo é totalmente utilizado.

\section{Trabalhos Relacionados}

O surgimento de redes ópticas elásticas com multiplexação espacial tem motivado várias investigações, principalmente em algoritmos de roteamento e alocação de espectro e núcleo, mas apenas recentemente esquemas de proteção foram propostos.

Em [Saridis et al. 2015], analisou-se o progresso da pesquisa em fibras de multiplexação por divisão espacial e os componentes de rede. Eles introduziram duas figuras visando a avaliação quantitativa de tecnologias, como amplificadores, multiplexadores, transmissores, switches e nós SDM.

Os autores em [Moura and da Fonseca 2016], introduziram um algoritmo de RCSA com base no algoritmo CCL (Connected Component Labelling). Neste artigo. políticas de ajuste do espectro são propostas para serem empregadas em conjunto com o algoritmo CCL.

Em [Oliveira and da Fonseca 2016a] introduziram um algoritmo baseado em pcycle para fornecer proteção de caminho independente de falhas para redes ópticas elásticas com multiplexação de divisão espacial.

em [Oliveira and da Fonseca 2016b]

$\mathrm{e}$

[Oliveira and da Fonseca 2017b], propõe-se um algoritmo para fornecer proteção utilizando p-cycle FIPP e mínima interferência em redes ópticas elásticas usando multiplexação por divisão espacial.

Hirota et.al. [Tode and Hirota 2014] o problema de RSCA é dividido em problema de roteamento e SCA. Neste artigo é introduzido um método de pré-computação de $k$ caminhos como uma solução de roteamento.

Em [Tan et al. 2016] foi proposto o algoritmo Cap-DPP que utiliza caminhos dedicados para realizar proteção, e utiliza uma técnica de $k$ caminhos para encontrar o caminho primário e o caminho de backup.

No [Oliveira and d. Fonseca 2017], propõe-se um algoritmo para fornecer proteção usando p-cycle FIPP e modulação. Os autores avaliaram a eficiência energética do algoritmo combinando p-cycle e modulação adaptativa.

Os autores no [Khodashenas et al. 2016] avaliaram as vantagens de usar a dimensão extra introduzida pela multiplexação por divisão espacial (SDM) para fins de alocação dinâmica de largura de banda em uma rede óptica flexível.

A sobreposição de espectro e o p-cycle FIPP foram estudados em [Oliveira and da Fonseca 2016c] para fornecer proteção em redes ópticas elásticas. 


\section{O Algoritmo SBRC}

O algoritmo introduzido nesta subseção, chamado Spectrum, Backup, Routing and Core assignment (SBRC), decide sobre a provisão de proteção dos caminhos ópticos através do uso de caminhos de backup compartilhados utilizando agregação de tráfego e sobreposição de espectro. Neste artigo, assume-se que um caminho óptico é estabelecido se e somente se ele puder ser protegido por caminho de backup compartilhado. $\mathrm{O}$ algoritmo SBRC estende o algoritmo SBPPMC [Oliveira and da Fonseca 2017a], adicionando agregação de tráfego e sobreposição de espectro, o que proporciona alta eficiência espectral e reduzindo o custo da rede.

O algoritmo proposto modela a disponibilidade de espectro na rede como um multigrafo rotulado (Fig. 2.1) [Oliveira and da Fonseca 2016a]. Um rótulo em uma aresta representa a disponibilidade de um slot. Na Fig. 2.2, o multigrafo é dividido em $C$ multígrafos, onde $C$ é o número de núcleos. Cada um desses multígrafos é transformado em multígrafos com $N-b+1$ arestas, (Fig. 2.3) onde $b$ é a demanda de largura de banda no slot. Então, cada um desses multígrafos é transformado em $N-b+1$ grafos. Em outras palavras, o multigrafo original (Fig. 2.3) é transformado em $C \times(N-b+1)$ grafos (Fig. 2.4). Cada aresta desses grafos representa uma combinação de $b$ slots. Esta representação assegura a contiguidade do espectro à solução. Nesses grafos, (Fig. 2.4), um valor de $\infty$ significa que pelo menos um slot já está alocado enquanto um valor menor significa que todos os slots estão disponíveis para alocação.

A seguinte notação será usada para descrever o algoritmo:

$s:$ nó fonte;

$d:$ nó destino;

$b$ : número de slots requisitados considerando agregação de tráfego;

$F$ : número de enlaces físicos;

$N$ : número de slots entre dois nós;

$C:$ número de núcleos entre dois nós;

$V:$ conjunto de nós; nós $u$ e $v$;

$e_{u, v, n}$ : a n-ésima aresta conectando $u$ e $v$. Cada aresta representa um slot entre os

$E=\left\{e_{u, v, n}\right\}:$ conjunto de arestas;

$G=(V, E, W)$ : multigrafo marcado composto por um conjunto de nós $V$, um conjunto de arestas $E$ e um conjunto de pesos de arestas $W,|E|=C \cdot N \cdot F$;

$r(s, d, b)$ : requisição do nó $s$ para o nó $d$ com demanda $b$;

$w\left(e_{u, v, n}\right)$ : peso da aresta $e_{u, v, n} ; w\left(e_{u, v, n}\right)<\infty$ se o n-ésimo slot no enlace conectando os OXC $u$ e $v$ estão livre e $w\left(e_{u, v, n}\right)=\infty$ se o slot já esta alocado;

$W=\left\{w\left(e_{u, v, n}\right)\right\}:$ conjunto de peso das arestas;

$\tau(G, C, b)=\left\{\widetilde{G}_{n, b}\right\}:$ função que produz todos os $\sigma$ grafos de $G ;$

$\sigma=\left|\left\{\widetilde{G}_{n, b}\right\}\right|=C \times(N-b+1)$ : número de grafos extraído do multigrafo;

$\widetilde{V}=V$ : conjunto de nós dos grafos gerados; 


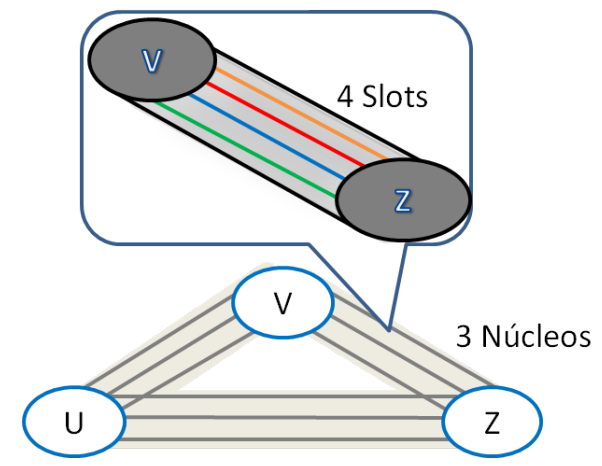

2.1 Exemplo de rede com 3 núcleos e 4 slots.

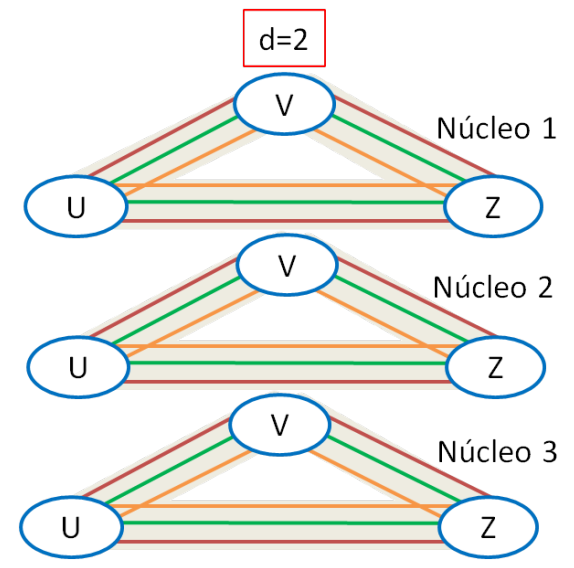

2.3 O Multigrafo em que um conjunto de arestas são mapeadas para uma aresta, restrição de contiguidade.
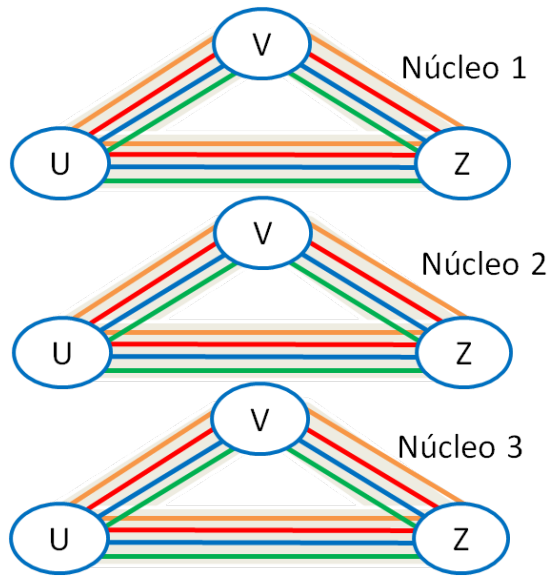

2.2 O Multigrafo, separado por núcleos, cada núcleo com 4 slots.

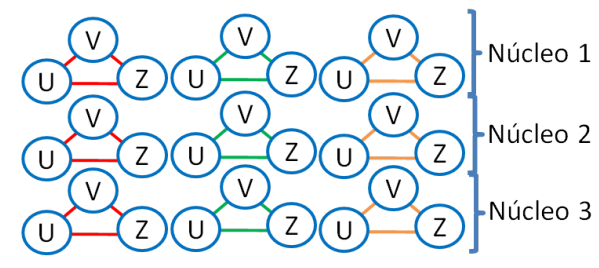

2.4 Grafos gerados.

Figura 2. Transformando multigrafo em grafos

$\widetilde{e}_{\tilde{u}, \widetilde{v}}=\left\{e_{u, v, n}\right\} \in E$ é uma sequencia tal que $e_{u, v, n}$ é a menor aresta ordenada, $e_{u, v, n+b}$ é a maior aresta ordenada e $\left|\widetilde{e}_{u, v}\right|=b$;

$\widetilde{w}_{n}\left(\widetilde{e}_{\widetilde{u}, \widetilde{v}}\right):$ peso da aresta $\widetilde{e}_{\widetilde{u}, \tilde{v}} ;$

$\widetilde{W}=\widetilde{w}_{n}\left(\widetilde{e}_{\widetilde{u}, \tilde{v}}\right)$;

$\widetilde{G}_{n, b}=(\widetilde{V}, \widetilde{E}, \widetilde{W})$ : o n-ésimo grafo marcado tal que $\widetilde{E}$ é o conjunto de arestas conectando $\{\widetilde{u}, \widetilde{v}\} \in \widetilde{V}$ e $\widetilde{W}$ é conjunto de custos associados a $\widetilde{E}$. As arestas em $\widetilde{E}$ correspondem ao mapeamento de $b$ arestas em $G$ iniciando na n-ésima aresta;

$\delta(G, r(s, d, b))$ : menor caminho entre $s$ e $d$ em $G$ que satisfazem a requisição de $b$ slots ;

$P_{n}$ : sequência de $\widetilde{G}_{n, b}$ tal que o nó fonte $s$ é o menor nó ordenado e $d$ é o maior nó ordenado;

$W\left(P_{n}\right): \sum_{\widetilde{e} \widetilde{u}, v} \in\left\{P_{n}\right\} \widetilde{e}_{\widetilde{u}, \widetilde{v}}$ : o peso do caminho $P_{n}$ (a soma dos pesos de todas as arestas de um caminho); 
$W_{P_{s, d}}=$ peso do menor caminho entre $s$ e $d$;

$L_{u, v}$ : conjunto de todos os caminhos de backup entre $u$ e $v$ em $G$;

$\rho\left(P_{n}, L_{u, v}, r(s, d, b)\right)$ : o menor caminho de backup em $L_{u, v}$ que $P_{L_{u, v}}$ são enlaces disjuntos de $P_{n}$ e satisfazem a requisição de banda $b$;

$\kappa\left(G, C, b, P_{n}\right)=\left\{\widetilde{G}_{n, b}\right\}$ : função que produz todos os grafos de $G$, considerando que os slots de proteção podem ser compartilhados, desde que os caminhos de trabalho $\left(P_{n}\right)$ que são protegidos pelos caminhos de backup que usam o slot sejam fisicamente disjuntas (compartilhamento de espectro); ordenado;

$L_{n}$ : sequência de $\widetilde{G}_{n, b}$ tal que o nó $s$ é o menor nó ordenado e $d$ é o maior nó

$P_{L_{u, v}}$ : conjunto de todos os caminhos protegidos pelo caminho de backup $L_{u, v}$;

$H_{P_{n}}$ : conjunto de todos os slots usado pelo caminho $P_{n}$;

$H_{P_{L_{u}, v}}$ : conjunto de todos os slots usados por todos os caminhos protegidos pelo caminho de backup $L_{u, v}$;

$L=\left\{L_{u, v}\right\}$ : conjunto de todos os caminhos de backup estabelecidos;

$\zeta\left(\widetilde{G}_{n, b_{m}}, P_{n}, r(s, d, b)\right)$ : menor caminho entre $s$ e $d$ em $\widetilde{G}_{n, b}$, considerando que $H_{P_{L_{u, v}}}$ é disjunto de $H_{P_{n}}$;

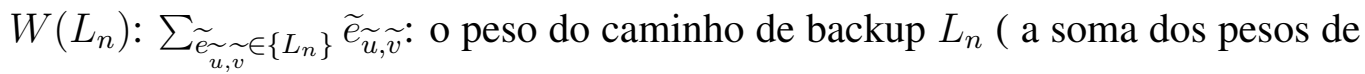
todas as arestas na sequencia);

$W_{L_{s, d}}=$ peso do caminho de backup que protege o caminho entre $s$ e $d$;

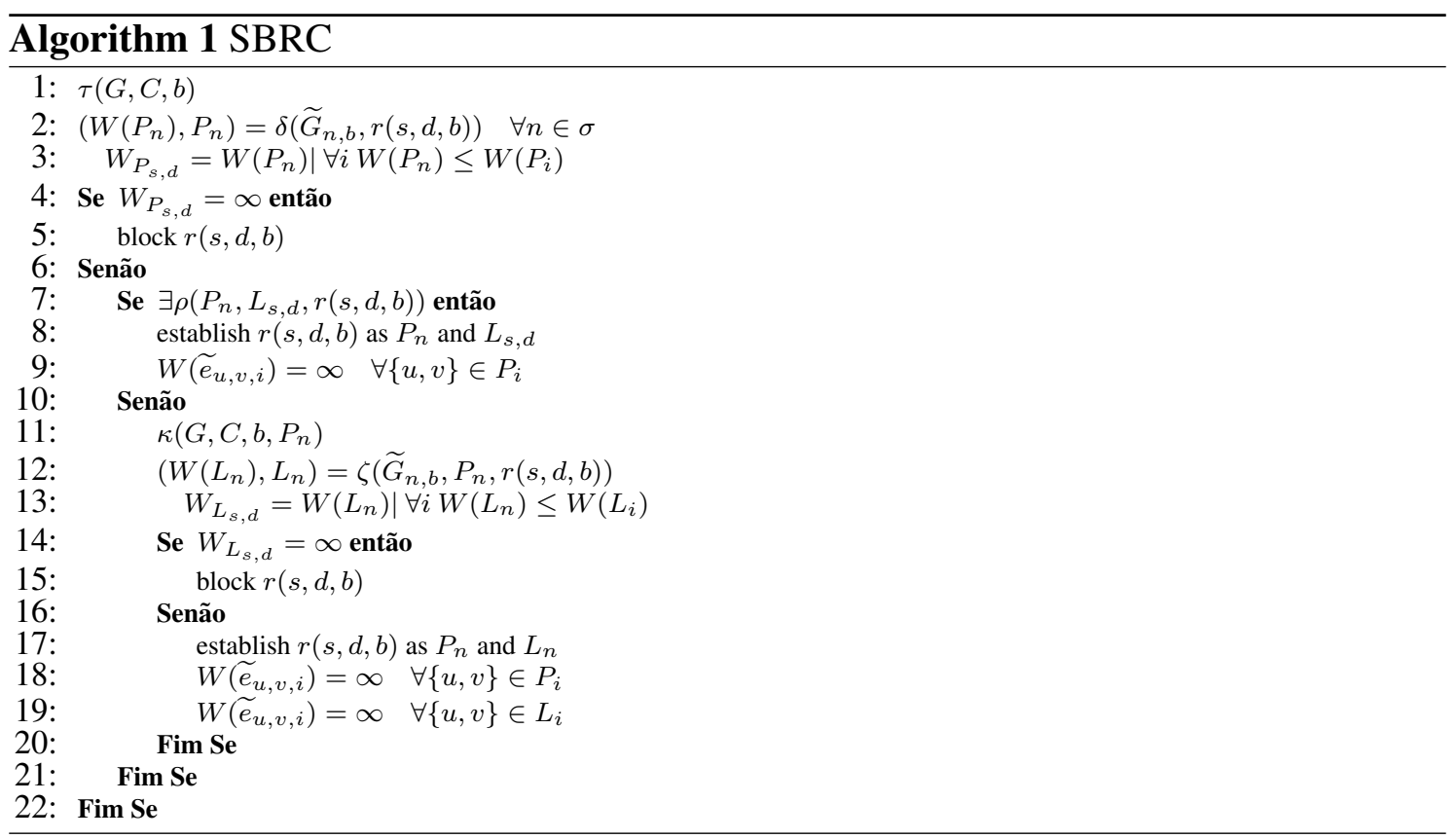

No algoritmo SBRC (Algoritmo 1) a linha 1 transforma o multigrafo em $C \times$ $(N-b+1)$ grafos, deste modo facilitando a busca de caminhos nos grafos gerados. A busca do caminho primário (linha 2) é realizado utilizando o algoritmo de Dikjstra para os grafos gerados, sendo escolhido o caminho com menor custo (linha 3). Se não for 
possível encontrar um caminho que respeite a restrições de contiguidade e continuidade para a demanda $b$ (linha 4), então a conexão é bloqueada (linha 5). Caso contrario, um caminho de backup será buscado dentre os caminhos de backup já estabelecidos para outras requisições. Se existir um caminho de backup disponível (linha 7), a requisição é estabelecida (linha 8) e as arestas correspondentes no multigrafo $G$ terão seus pesos configurados para $\infty$ (linha 9), significando que os slots foram alocados para o caminho. Se não existir nenhum caminho de backup que possa proteger a requisição, então um novo caminho precisa ser criado. Na linha 11, o multigrafo é transformado em $C \times(N-b)$ grafos, considerando a agregação de trafego, ou seja, a não reserva de banda de guarda e a sobreposição de slots, ou seja, slots que estão protegendo outras conexões podem ser utilizados desde que respeitando as regras de compartilhamento. Os grafos gerados consideram ainda que enlaces do caminho primário estão reservados. A linha 12 utiliza o algoritmo de Dikjstra e os grafos gerados na linha 11 para buscar um menor caminho de backup nos grafos. A linha 13 escolhe o menor caminho dos grafos para caminho de backup. Se o peso do menor caminho for $\infty$, significa que um caminho de proteção não pode ser encontrado e a requisição é bloqueada (linha 15). Caso contrario linha 17, o caminho primário bem como o caminho de backup são estabelecidos e as correspondentes arestas no multigrafo $G$ tem seus pesos configurados para $\infty$ (linhas 18 e 19).

A complexidade de transformar o multigrafo em grafos é $O(E+V)$. Para encontrar o caminho primário, no pior caso, o algoritmo de Dijkstra é executado em $C \times(N-b)$, onde a complexidade amortizada de Dijkstra é $O(E+V \log V)$. Da mesma forma, para o caminho de backup, a complexidade de transformar o multigrafo em grafos é $O(E+V)$, considerando a agregação e a sobreposição de espectro; em seguida o algoritmo de Dijkstra é executado em $C \times(N-b)$ grafos. Logo, no pior caso, o algoritmo SBRC tem complexidade de $2 \times(E+V+C \times(N-b) \times(E+V \log V))$. Como $N, C$ e $b$ podem ser expressados como constantes, a complexidade do algoritmo é $O(E+V \log V)$.

\section{Avaliação de Desempenho}

Para avaliar o desempenho do Algoritmo SBRC, experimentos de simulação foram realiza- dos utilizando o simulador FlexGridSim [Moura and Drummond ]. Em cada simulação, 100.000 requisições foram geradas. Utilizou-se o método de replicação independente e adotou-se nível de confiança de $95 \%$ para os intervalos de confiança. As topologias NSF (Figura 3.2) com 14 nós e 18 enlaces bidirecionais e a topologia USA (Figura 3.2) com 24 nós e 43 enlaces bidirecionais foram empregadas, as figuras mostram as respectivas distancias entre os nós. Variou-se a carga entre 25 e 400 erlangs em intervalos de 25 erlangs. Foram utilizados sete tipos de requisições de $25 \mathrm{Gbps}, 50 \mathrm{Gbps}$, 125 Gbps, $200 \mathrm{Gbps}, 500 \mathrm{Gbps}, 750 \mathrm{Gbps}$ e 1 Tbps. Os enlaces foram compostos por fibras com 7 núcleos e cada núcleo foi dividido em 320 slots. As métricas utilizadas na comparação são relação de bloqueio de banda e relação de crosstalk por slot.

Nas figuras, as curvas rotuladas como SBPPMC mostram os resultados para as redes que utilizam o algoritmo SBPPMC [Oliveira and da Fonseca 2017a], as curvas rotuladas como SSCA encontra o caminho primário através do algoritmo proposto em [Tode and Hirota 2014], utilizando $k=3$ e o caminho de proteção adicionando a capacidade de compartilhamento ao mesmo algoritmo, as curvas rotuladas como Cap-DPP mostram os resultados para as redes que utilizam o algoritmo proposto em [Tan et al. 2016], enquanto as curvas rotuladas como SBRC mostram os resultados para as redes que utilizam o algoritmo proposto neste artigo. 


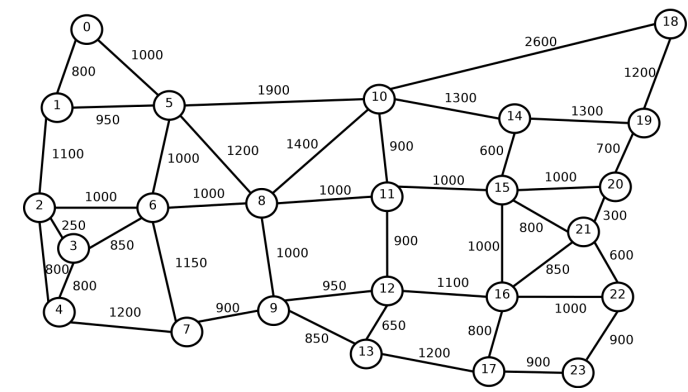

3.1 Topologia USA

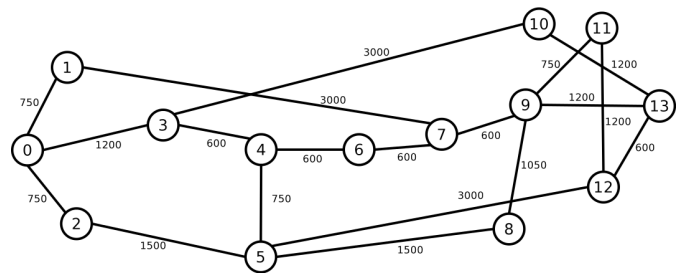

3.2 Topologia NSF

Figura 3. Topologias

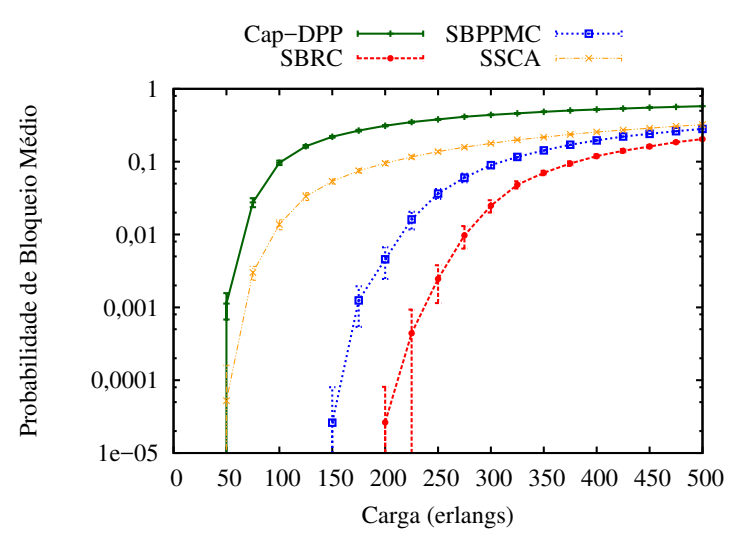

4.1 USA

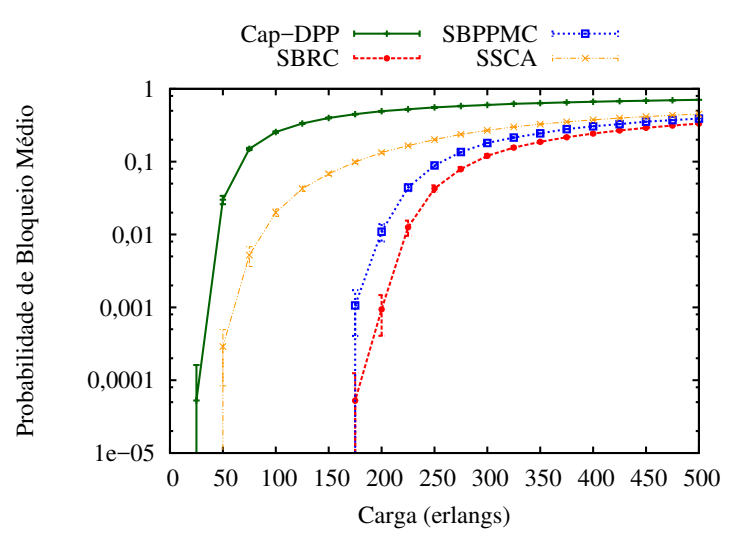

4.2 NSF

Figura 4. Bloqueio de banda em função da carga da rede

As Figuras 4.1 e 4.2 mostram a probabilidade de bloqueio (Bandwidth Blocking Ratio) para a topologia USA e NSF, respectivamente.

Para Fig. 4.1 enquanto o Cap-DPP e o SSCA começam a bloquear requisições sob cargas de 50 erlangs, o SBPPMC começa a bloquear apenas sob cargas de 125 erlangs. O SBRC por sua vez inicia o bloqueio de requisições somente sob cargas de 200 erlangs. O alto número de requisições bloqueadas para o algoritmo Cap-DPP é consequencia do não compartilhamento de recursos para a criação do caminho de backup, gerando maior utilização e bloqueando futuras requisições. Apesar do algoritmo SSCA realizar compartilhamento de recursos do caminho de backup a técnica de geração dos caminhos possibilita uma maior fragmentação gerando um maior bloqueio de requisições do que os algoritmos que utilizam o multigrafo. Embora o SBRC e o SBPPMC utilizem o multigrafo, a maior BBR do algoritmo SBPPMC é produzida devido a não utilização de agregação de tráfego e compartilhamento de espectro, técnicas utilizadas pelo algoritmo SBRC.

Para Fig. 4.2 enquanto o SBPPMC e o SBRC começam a bloquear requisições sob cargas de 200 erlangs, o Cap-DPP começa a bloquear sob cargas de 25 erlangs, e o SBRC por sua vez inicia o bloqueio de requisições somente sob cargas de 50 erlangs. Devido a menor conectividade da topologia NSF os algoritmos geram bloqueios com menor carga. Como na topologia USA, a BBR alta produzida pelo Cap-DPP é uma consequência de não compartilhar caminhos de backup. Além disso, o Cap-DPP e o SSCA produziram altos 
valores de BBR, porque eles não utilizam a representação do espectro em multigrafo, que permite que as decisões sejam feitas considerando uma granularidade de alocação de espectro fino, evitando assim a fragmentação do espectro. Por sua vez o algoritmo SBRC gerou valores de BBR apenas $10 \%$ menor que o SBPPMC evidenciando que a utilização de agregação de trafego e sobreposição de espectro produz melhores resultados com topologias com maior conectividade.

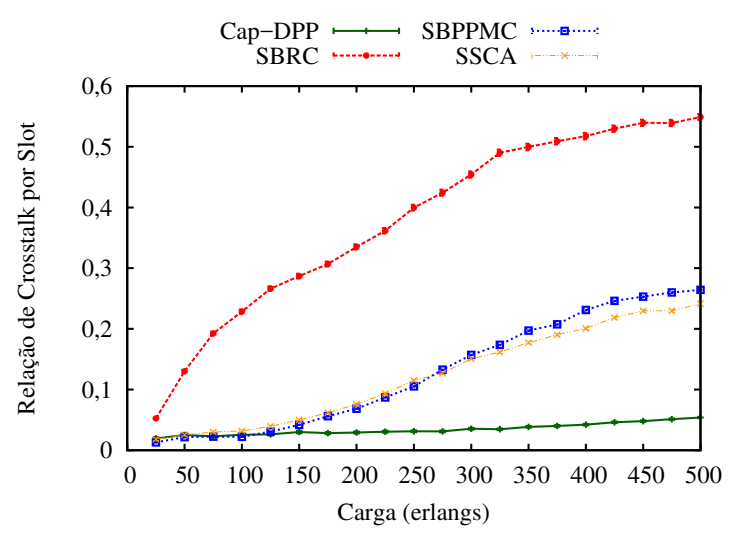

5.1 USA

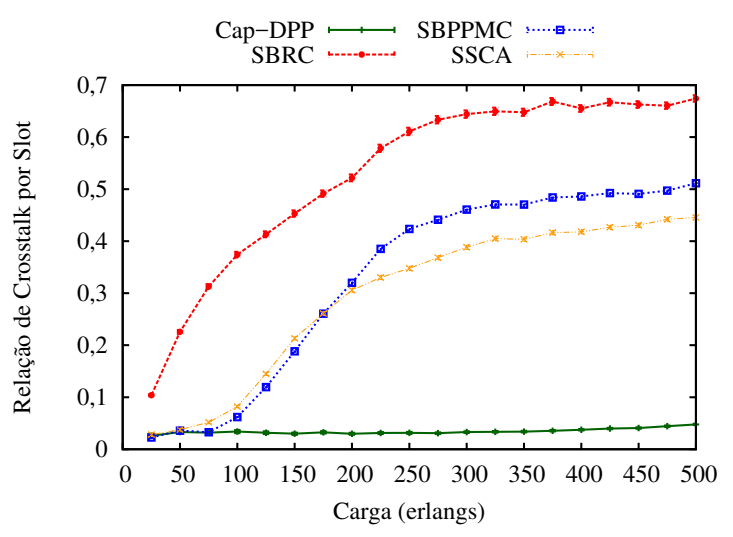

$5.2 \mathrm{NSF}$

Figura 5. Relação de crosstalk por slot

As Figuras 5.1 e 5.2 representam a relação de crosstalk por slot em função da carga da rede para as topologia USA e NSF, respectivamente. O valor de crosstalk para cada slot de espectro é definido como a razão entre o índice de crosstalk atual e o valor máximo do índice crosstalk. A relação de crosstalk por slot é definida pelo valor médio entre todos os slots de espectro da rede [Fujii et al. 2014].

$\mathrm{Na}$ Fig. 5.1, o CpS gerado pelo algoritmo SBRC inicia em 0,05 e aumenta até 0,54 enquanto que os outros algoritmos SBPPMC e SSCA iniciam em 0,02 e aumentam até 0,27 . O CpS gerado para o algoritmo Cap-DPP começa em 0,01 e aumenta até 0,05. $\mathrm{O}$ algoritmo Cap-DPP produz os valores $\mathrm{CpS}$ mais baixos, como consequência do alto bloqueio e baixa utilização produzida. O algoritmo SBRC produziu maior $\mathrm{CpS}$ que os outros algoritmos isso ocorre devido ao maior número de requisições aceitas. Além disso a utilização de sobreposição de espectro beneficia uma melhor fragmentação, o que pode ocasionar um maior $\mathrm{CpS}$.

$\mathrm{Na}$ Fig. 5.2, o $\mathrm{CpS}$ gerado pelo algoritmo SBRC inicia em 0,1 e aumenta até 0,67 enquanto que os outros algoritmos SBPPMC e SSCA iniciam em 0,03 e aumentam até 0,51 e 0,44 , respectivamente. O CpS gerado para o algoritmo Cap-DPP inicia em 0,03 e aumenta até 0,05. O algoritmo Cap-DPP produz os valores $\mathrm{CpS}$ mais baixos, como consequência do alto bloqueio e baixa utilização produzida. Apesar da BBR dos algoritmos SBRC e SBPPMC serem bem próximas, o $\mathrm{CpS}$ do algoritmo SBRC é maior, pois a agregação de tráfego e a sobreposição de espectro geram em um maior numero de conexões utilizando slots adjacentes, gerando maiores valores de $\mathrm{CpS}$.

\section{Conclusão}

Este artigo introduziu um algoritmo para resolver os problemas de proteção e roteamento e alocação de núcleo e espectro. Foi proposto uma nova abordagem para apoiar o estabelecimento de caminhos de luz em redes ópticas elásticas de multiplexação por divisão 
espacial protegidas por caminhos de backup compartilhados usando agregação de tráfego óptica e sobreposição de espectro. O algoritmo foi avaliado para diferentes topologias e cargas. O SBRC foi comparado a outros algoritmos da literatura. Os resultados da simulação mostram o melhor desempenho do algoritmo SBRC quando comparado aos outros algoritmos avaliados.

\section{Agradecimento}

O presente trabalho foi realizado com apoio do Conselho Nacional de Desenvolvimento Científico e Tecnológico - Brasil (CNPq), processo número 165446/2015-3 e da Fundação de Amparo à Pesquisa do Estado de São Paulo (FAPESP), processo número 15/24494-8.

\section{Referências}

Fujii, S., Hirota, Y., Tode, H., and Murakami, K. (2013). On-demand spectrum and core allocation for multi-core fibers in elastic optical network. In Optical Fiber Communication Conference and Exposition and the National Fiber Optic Engineers Conference (OFC/NFOEC), 2013, pages 1-3.

Fujii, S., Hirota, Y., Tode, H., and Murakami, K. (2014). On-demand spectrum and core allocation for reducing crosstalk in multicore fibers in elastic optical networks. Optical Communications and Networking, IEEE/OSA Journal of, 6(12):1059-1071.

Grüner-Nielsen, L., Sun, Y., Nicholson, J. W., Jakobsen, D., Jespersen, K. G., R. Lingle, J., and Pálsdóttir, B. (2012). Few mode transmission fiber with low dgd, low mode coupling, and low loss. J. Lightwave Technol., 30(23):3693-3698.

Hayashi, T., Taru, T., Shimakawa, O., Sasaki, T., and Sasaoka, E. (2011). Design and fabrication of ultra-low crosstalk and low-loss multi-core fiber. Opt. Express, 19(17):16576-16592.

Hayashi, T., Taru, T., Shimakawa, O., Sasaki, T., and Sasaoka, E. (2012). Uncoupled multi-core fiber enhancing signal-to-noise ratio. Opt. Express, 20(26):B94-B103.

Huang, H., Huang, S., Yin, S., Zhang, M., Zhang, J., and Gu, W. (2016). Virtual network provisioning over space division multiplexed optical networks using few-mode fibers. IEEE/OSA Journal of Optical Communications and Networking, 8(10):726-733.

Ito, T., de Gabory, E. L. T., Arikawa, M., Hashimoto, Y., and Fukuchi, K. (2013). Reduction of influence of inter-core cross-talk in mcf with bidirectional assignment between neighboring cores. In Optical Fiber Communication Conference/National Fiber Optic Engineers Conference 2013, page OTh3K.2. Optical Society of America.

Khodashenas, P. S., Rivas-Moscoso, J. M., Siracusa, D., Pederzolli, F., Shariati, B., Klonidis, D., Salvadori, E., and Tomkos, I. (2016). Comparison of spectral and spatial super-channel allocation schemes for sdm networks. Journal of Lightwave Technology, 34(11):2710-2716.

Koshiba, M., Saitoh, K., Takenaga, K., and Matsuo, S. (2011). Multi-core fiber design and analysis: coupled-mode theory and coupled-power theory. Opt. Express, 19(26):B102B111.

Liu, M., Tornatore, M., and Mukherjee, B. (2013). Survivable traffic grooming in elastic optical networks -shared protection. Lightwave Technology, Journal of, 31(6):62306234. 
Mori, T., Sakamoto, T., Wada, M., Yamamoto, T., and Yamamoto, F. (2014). Six-lp-mode transmission fiber with dmd of less than $70 \mathrm{ps} / \mathrm{km}$ over c+l band. In $O F C 2014$, pages $1-3$.

Moura, P. M. and da Fonseca, N. L. S. (2016). Routing, core and spectrum assignment based on connected component labelling for sdm optical networks. In 2016 IEEE International Conference on Communications (ICC), pages 1-6.

Moura, P. M. and Drummond, A. C. FlexGridSim: Flexible Grid Optical Network Simulator. http://www.lrc.ic.unicamp.br/FlexGridSim/.

Muhammad, A., Zervas, G., Saridis, G., Salas, E., Simeonidou, D., and Forchheimer, R. (2014a). Flexible and synthetic sdm networks with multi-core-fibers implemented by programmable roadms. In Optical Communication (ECOC), 2014 European Conference on, pages $1-3$.

Muhammad, A., Zervas, G., Simeonidou, D., and Forchheimer, R. (2014b). Routing, spectrum and core allocation in flexgrid sdm networks with multi-core fibers. In Optical Network Design and Modeling, 2014 International Conference on, pages 192-197.

Oliveira, H. M. N. S. and d. Fonseca, N. L. S. (2017). Proteção de redes Ópticas elásticas com multiplexação espacial baseada em modulação, p-cycle FIPP e interferência mínima. In 2017 Brazilian Symposium on Computer Networks and Distributed Systems, pages 831-844.

Oliveira, H. M. N. S. and da Fonseca, N. L. S. (2016a). Algorithm for protection of space division multiplexing elastic optical networks. In 2016 IEEE Global Communications Conference (GLOBECOM), pages 1-6.

Oliveira, H. M. N. S. and da Fonseca, N. L. S. (2016b). The minimum interference p-cycle algorithm for protection of space division multiplexing elastic optical networks. In 2016 8th IEEE Latin-American Conference on Communications (LATINCOM), pages $1-6$.

Oliveira, H. M. N. S. and da Fonseca, N. L. S. (2016c). Traffic grooming and spectrum overlap in fipp p-cycle for protection of elastic optical networks. In 2016 8th IEEE Latin-American Conference on Communications (LATINCOM), pages 1-6.

Oliveira, H. M. N. S. and da Fonseca, N. L. S. (2017a). Algorithm for shared path for protection of space division multiplexing elastic optical networks. In 2017 IEEE International Conference on Communications (ICC), pages 1-6.

Oliveira, H. M. N. S. and da Fonseca, N. L. S. (2017b). The minimum interference pcycle algorithm for protection of space division multiplexing elastic optical networks. IEEE Latin America Transactions, 15(7):1342-1348.

Oliveira, H. M. N. S. and da Fonseca, N. L. S. (2017c). Proteção de redes opticas elásticas com multiplexação espacial baseada em modulação, p-cycle fipp e interferência minima. In XXXV Brazilian Symposium on Computer Networks and Distributed Systems 2017, pages $1-14$.

Proietti, R., Liu, L., Scott, R., Guan, B., Qin, C., Su, T., Giannone, F., and Yoo, S. (2015). 3d elastic optical networking in the temporal, spectral, and spatial domains. Communications Magazine, IEEE, 53(2):79-87. 
Ryf, R., Essiambre, R. J., Randel, S., Mestre, M. A., Schmidt, C., and Winzer, P. J. (2012). Impulse response analysis of coupled-core 3-core fibers. In 2012 38th European Conference and Exhibition on Optical Communications, pages 1-3.

Sakaguchi, J., Puttnam, B. J., Klaus, W., Awaji, Y., Wada, N., Kanno, A., Kawanishi, T., Imamura, K., Inaba, H., Mukasa, K., Sugizaki, R., Kobayashi, T., and Watanabe, M. (2013). $305 \mathrm{tb} / \mathrm{s}$ space division multiplexed transmission using homogeneous 19-core fiber. J. Lightwave Technol., 31(4):554-562.

Saridis, G. M., Alexandropoulos, D., Zervas, G., and Simeonidou, D. (2015). Survey and evaluation of space division multiplexing: From technologies to optical networks. IEEE Communications Surveys Tutorials, 17(4):2136-2156.

Sillard, P., Molin, D., Bigot-Astruc, M., Maerten, H., Ras, D. V., and Achten, F. (2014). Low-dmgd 6-lp-mode fiber. In OFC 2014, pages 1-3.

Takara, H., Sano, A., Kobayashi, T., Kubota, H., Kawakami, H., Matsuura, A., Miyamoto, Y., Abe, Y., Ono, H., Shikama, K., Goto, Y., Tsujikawa, K., Sasaki, Y., Ishida, I., Takenaga, K., Matsuo, S., Saitoh, K., Koshiba, M., and Morioka, T. (2012). 1.01-pb/s (12 $\mathrm{sdm} / 222 \mathrm{wdm} / 456 \mathrm{gb} / \mathrm{s}$ ) crosstalk-managed transmission with $91.4-\mathrm{b} / \mathrm{s} / \mathrm{hz}$ aggregate spectral efficiency. In European Conference and Exhibition on Optical Communication, page Th.3.C.1. Optical Society of America.

Takenaga, K., Arakawa, Y., Sasaki, Y., Tanigawa, S., Matsuo, S., Saitoh, K., and Koshiba, M. (2011). A large effective area multi-core fiber with an optimized cladding thickness. Opt. Express, 19(26):B543-B550.

Tan, Y., Zhu, R., Yang, H., Zhao, Y., Zhang, J., Liu, Z., Qu, Q., and Zhou, Z. (2016). Crosstalk-aware provisioning strategy with dedicated path protection for elastic multicore fiber networks. In 2016 15th International Conference on Optical Communications and Networks (ICOCN), pages 1-3.

Tode, H. and Hirota, Y. (2014). Routing, spectrum and core assignment for space division multiplexing elastic optical networks. In 2014 16th International Telecommunications Network Strategy and Planning Symposium (Networks), pages 1-7.

Winzer, P., Gnauck, A., Konczykowska, A., Jorge, F., and Dupuy, J.-Y. (2011). Penalties from in-band crosstalk for advanced optical modulation formats. In 37th European Conference and Exposition on Optical Communications, page Tu.5.B.7. Optical Society of America.

Zhang, G., De Leenheer, M., and Mukherjee, B. (2012). Optical traffic grooming in ofdmbased elastic optical networks [invited]. Optical Communications and Networking, IEEE/OSA Journal of, 4(11):B17-B25. 